\title{
A system for the measurement of gene targeting efficiency in human cell lines using an antibiotic resistance-GFP fusion gene
}

Yuko Konishi*, Sivasundaram Karnan*, Miyuki Takahashi, Akinobu Ota, Lkhagvasuren Damdindorj, Yoshitaka Hosokawa, and Hiroyuki Konishi

Department of Biochemistry, Aichi Medical University School of Medicine, Nagakute, Aichi, Japan

BioTechniques 53:141-152 (September 2012) doi 10.2144/0000113911

Keywords: gene editing; genome modification; homologous recombination; adeno-associated virus

${ }^{*}$ Y.K. and S.K. contributed equally to this work.

Gene targeting in a broad range of human somatic cell lines has been hampered by inefficient homologous recombination. To improve this technology and facilitate its widespread application, it is critical to first have a robust and efficient research system for measuring gene targeting efficiency. Here, using a fusion gene consisting of hygromycin B phosphotransferase and 3'-truncated enhanced GFP ( $\left.\mathrm{Hyg}^{\mathrm{R}}-5^{\prime} \mathrm{EGFP}\right)$ as a reporter gene, we created a molecular system monitoring the ratio of homologous to random integration $(\mathrm{H} / \mathrm{R}$ ratio) of targeting vectors into the genome. Cell clones transduced with a reporter vector containing $\mathrm{Hyg}^{\mathrm{R}}-5^{\prime}$ EGFP were efficiently established from two human somatic cell lines. Established $\mathrm{Hyg}^{\mathrm{R}}-5^{\prime}$ EGFP reporter clones retained their capacity to monitor gene targeting efficiency for a longer duration than a conventional reporter system using an unfused $5^{\prime}$ EGFP gene. With the $\mathrm{Hyg}^{\mathrm{R}}-5^{\prime}$ EGFP reporter system, we reproduced previous findings of gene targeting frequency being up-regulated by the use of an adeno-associated viral (AAV) backbone, a promoter-trap system, or a longer homology arm in a targeting vector, suggesting that this system accurately monitors $\mathrm{H} / \mathrm{R}$ ratio. Thus, our $\mathrm{Hyg}^{\mathrm{R}}-5^{\prime} \mathrm{EGFP}$ reporter system will assist in the development of an efficient AAV-based gene targeting technology.

Gene targeting in human somatic cells is a powerful technologyallowing for the biological analyses of human gene function in physiological settings. Gene targeting is achieved by homologous recombination (HR) between the endogenous gene loci and targeting vectors introduced into cells. The use of gene-targeted human cell systems is particularly advantageous for the study of genetic changes leading to human diseases such as cancer $(1,2)$. These cell systems are also expected to serve as a platform for compound screening and thereby facilitate the development and validation of molecularly targeted therapies $(3,4)$.

Although gene targeting in human cells has improved significantly (5-9), the execution of gene targeting in human somatic cell lines continues to be labor-intensive and difficult to achieve depending on the genomic loci targeted and the cell lines used. This is probably because most somatic cells have low HR efficiency compared with embryonic stem cells as shown with murine myoblasts (10). To further improve this technology, it is critical to first have a robust and efficient research system to measure gene targeting efficiency in human somatic cell lines. In practice, the ratio of homologous to random integration $(\mathrm{H} / \mathrm{R}$ ratio) of targeting vectors should be closely monitored, as a high $\mathrm{H} / \mathrm{R}$ ratio is critical for efficient gene targeting.

In previous studies, monitoring of gene targeting efficiency has been performed using vectors carrying reporter genes such as enhanced GFP (EGFP), neomycin phosphotransferase $\left(\mathrm{Neo}^{\mathrm{R}}\right)$, and alkaline phosphatase genes that harbor intragenic inactivating mutations, deletions, or small insertions $(5,11,12)$. Cells are first transduced with these reportervectors and then with targetingvectors containing partial fragments of wild-type reporter genes that correct the inactivated reporter genes via $\mathrm{HR}$ and reconstitute intact reporter function. However, one concern with these reporter systems is unstable and insufficient expression of the reporter genes. Because reporter genes are generally driven by viral promoters, their expression tends to diminish over time probably because of epigenetic changes within the viral promoters that arise during the establishment and maintenance of cell clones (13-15).

In this study, we sought to overcome this problem by using hygromycin B phosphotransferase $\left(\mathrm{Hyg}^{\mathrm{R}}\right)$ fused with 3'-truncated EGFP ( $\mathrm{Hyg}^{\mathrm{R}}-5^{\prime}$ EGFP) as a reporter gene. If the $\mathrm{Hyg}^{\mathrm{R}}-5^{\prime}$ EGFP gene is epigenetically silenced in a fraction of a reporter cell clone, this fraction will be eliminated by hygromycin selection. To evaluate the utility of this $\mathrm{Hyg}^{\mathrm{R}}-5^{\prime}$ EGFP reporter system, we assayed the $\mathrm{H} / \mathrm{R}$ ratios of targeting vectors in human cell lines in several experimental settings.

\section{Materials and methods}

Vector construction

For the creation of the $\mathrm{Hyg}^{\mathrm{R}}-5^{\prime}$ EGFP reporter vector, the $5^{\prime}$ portion of EGFP $(500 \mathrm{bp})$ was amplified by PCR and ligated to an EcoRIBam HI site of pIRESneo3 (BD Biosciences, Franklin Lakes, NJ, USA). A BstXI-XbaI 
fragment containing a synthetic intron, internal ribosomal entry site (IRES), and $\mathrm{Neo}^{\mathrm{R}}$ was then deleted from the resulting plasmid, and the remaining fragment was bluntended and self-ligated. This plasmid was then digested with NheI and AgeI and ligated to the PCR-amplified $\mathrm{Hyg}^{\mathrm{R}}$ coding region with a Kozak consensus sequence (GCCACC) at its $5^{\prime}$ terminus, so that the $\mathrm{Hyg}^{\mathrm{R}}-5^{\prime}$ EGFP was translated in frame. Finally, the $3^{\prime}$ homology arm consisting of the internal portion of simian virus 40 (SV40) large $\mathrm{T}(1 \mathrm{~kb})$ was PCR-amplified and inserted into a BstZ17I site of the plasmid.

To construct ATG-less targeting vector (TV), pAAV-MCS (Agilent Technologies, Santa Clara, CA, USA) was digested with NotI, and the backbone containing two inverted terminal repeats was recovered. Multiple restriction enzyme sites, including the NotI and XhoI sites, were then incorporated into this backbone, destroying the original NotI sites and thus generating "modified pAAV-MCS.” Meanwhile, an EGFP coding region without an authentic start codon was PCR-amplified and inserted into an $X b a \mathrm{I}$ site of pSEPT (a gift from Dr. Fred Bunz) (16) in the forward direction. The resulting plasmid was cut with $N o t \mathrm{I}$ and $X h o \mathrm{I}$, and the isolated NotI-XhoI fragment containing ATG-less EGFP and a promoter-trap assembly was ligated to a NotI--XhoI site of the modified pAAV-MCS. The resulting plasmid was finally digested with $X h o \mathrm{I}$ and ligated to an XhoI fragment from the $\mathrm{Hyg}^{\mathrm{R}}-5^{\prime}$ EGFP reporter consisting of the internal portion of SV40 large T (i.e., the 3' homology arm of the $\mathrm{Hyg}^{\mathrm{R}}-5^{\prime}$ EGFP reporter).

For the construction of SV40p TV, the bovine growth hormone polyadenylation site [BGH poly (A)] derived from pcDNA3.1 (Life Technologies, Carlsbad, CA, USA) was first inserted at the EcoRV site of plasmid 5A (a gift from Dr. Ben H. Park), a platform vector to construct targeting vectors carrying a $N e o^{R}$ gene expressed by an SV40 early promoter (17). The resulting plasmid containing BGH poly $(\mathrm{A})$ in the forward direction was cleaved with $N h e \mathrm{I}$ and $X m n I$ to remove the backbone of the plasmid. The recovered fragment was then blunt-ended and ligated in the forward direction to a blunt-ended SpeI-EcoRV fragment from ATG-less TV containing a backbone and both of the homology arms.

For 3' EGFP TV, a fragment between two XbaI sites in ATG-less TV (i.e., the EGFP coding region without an authentic start codon) was replaced with a PCR-amplified 3 ' partial EGFP coding region (606 bp).

To produce the 5 ' EGFP reporter vector, the $5^{\prime}$ portion of EGFP (500 bp) with a cytomegalovirus promoter was recovered as a SpeI fragment derived from pEGFPx2 (a gift from Dr. Ben H. Park) and inserted into

Table 1. Establishment of reporter clones.

\begin{tabular}{|c|c|c|c|}
\hline Reporter gene & Cell line & Single cell clones screened & Functional clones established \\
\hline Hyg $^{\mathrm{R}}$ 5 $^{\prime}$ EGFP & HCT116 & 6 & $1(16.7 \%)$ \\
\hline & DLD-1 & 6 & $1(16.7 \%)$ \\
\hline & Total & 12 & $2(16.7 \%)^{\mathrm{a}}$ \\
\hline $5^{\prime}$ EGFP & HCT116 & 69 & $2(2.9 \%)^{\mathrm{a}}$ \\
\hline
\end{tabular}

aNo statistical significance, Fisher's exact test.

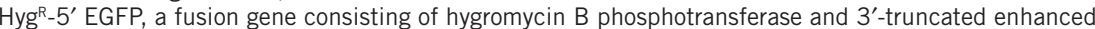
GFP; 5' EGFP, 3'-truncated enhanced GFP.

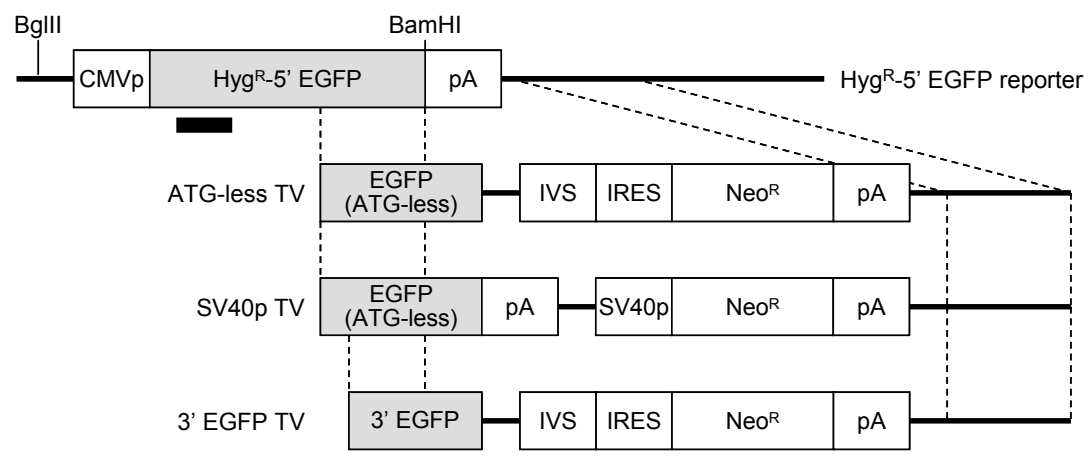

Figure 1. Representation of the reporter and targeting vectors. Vector names are denoted to the right (a reporter vector) or left (targeting vectors) of the diagrams. A filled rectangle indicates the location of a probe used for Southern blot analysis in Figure 2C. Bglll and BamHI sites on the Hyg ${ }^{\mathrm{R}}-5^{\prime}$ EGFP reporter vector that were cleaved during Southern blot analysis are marked in the diagram. HygR, hygromycin resistance gene; TV, targeting vector; CMVp, cytomegalovirus promoter; IVS, synthetic intron; IRES, internal ribosomal entry site; $\mathrm{NeO}^{\mathrm{R}}$, neomycin resistance gene; SV40p, simian virus

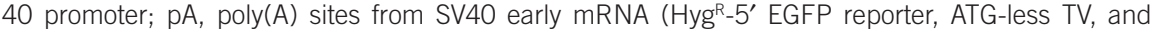
3' EGFP TV), the bovine growth hormone gene (SV40p TV, left), or a 49-bp-long synthetic poly(A) signal derived from pCl-neo (SV40p TV, right). Solid lines indicate vector sequences other than those specified above. Dotted lines indicate homologous regions between reporter and targeting vectors. For details on partial EGFP genes, see vector construction in the Materials and methods section. The diagrams are not drawn to scale.

pSEPT in the forward direction. To introduce a 3' homology arm, the resulting plasmid was cleaved with $X h o I$ and then ligated to the internal portion of SV40 large T excised from the $\mathrm{Hyg}^{\mathrm{R}}-5^{\prime}$ EGFP reporter vector. After transfection of the resulting plasmid into cells, stable transfectants were infected with an adenovirus encoding Cre, and a subclone undergoing Cre-loxP recombination was isolated from each stable clone; the $\mathrm{H} / \mathrm{R}$ ratio was then measured by infection with ATG-less TV. Throughout the construction of all plasmids described above, fragments amplified by PCR were confirmed by DNA sequencing after cloning into plasmids. The sequences of the plasmids have been deposited in GenBank (accession noumbers: $\mathrm{Hyg}^{\mathrm{R}}-5^{\prime}$ EGFP reporter, JQ394982; ATG-less TV,JQ394983; SV40p TV,JQ394984; 3' EGFPTV,JQ394985; and 5'EGFP reporter, JQ394986).

Transfection and infection

Cells were cultured in Dulbecco's modified Eagle's medium (DMEM; Wako Chemicals, Osaka, Japan) supplemented with $5 \%$ fetal bovine serum. Transfection of plasmids into cells was performed using TransIT-LT1 (Mirus Bio, Madison, WI, USA) as per the manufacturer's instructions. Adeno-associated viral
(AAV) particles (serotype 2) were produced by transfection of 293 T cells in $75 \mathrm{~cm}^{2}$ flasks with $7.5 \mu \mathrm{g}$ AAV-based targeting plasmids and the AAV Helper-Free system (Agilent Technologies). The copy numbers of the AAV particles were determined by real-time PCR performed with StepOnePlus (Life Technologies), using the solutions of the AAV-based targeting plasmids as controls. The copy numbers of the AAV-based targeting plasmids in the control samples were calculated using their molecular weights and concentrations measured by NanoDrop (NanoDrop Technologies, Wilmington, DE, USA).

Throughout the study, cells were infected with AAV particles as previously described (7) at a multiplicity of infection of $1 \times 10^{4}$. Selection with G418 (Life Technologies) was carried out at concentrations of 0.4 and 1 $\mathrm{mg} / \mathrm{mL}$ for the HCT116 and DLD-1 cell lines, respectively. In addition, 0.2 and 0.25 $\mathrm{mg} / \mathrm{mL}$ hygromycin (Wako Chemicals) were used for the selection of HCT116 and DLD-1 cells, respectively. For the assay of the $\mathrm{H} / \mathrm{R}$ ratio of the targeting vectors, reporter cell clones were infected with AAV-based targeting vectors in $75-\mathrm{cm}^{2}$ flasks, selected with G418 for 1-2 weeks, trypsinized, suspended in phosphate-buffered saline 
A

reporter:

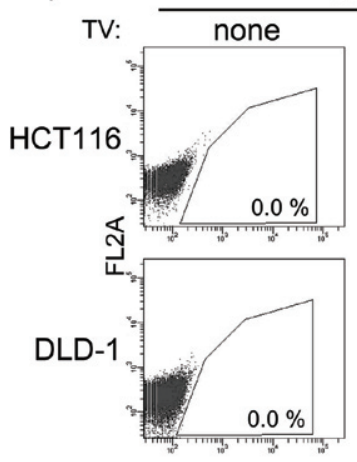

none

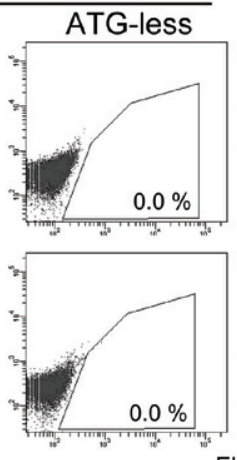

FL1A
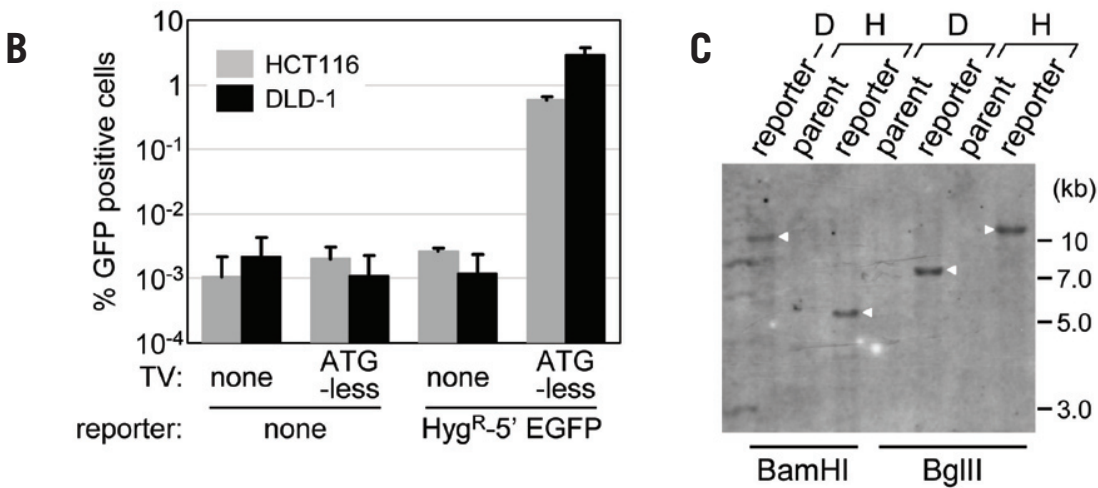

Figure 2. The Hyg ${ }^{\mathrm{R}}-5^{\prime}$ EGFP reporter system for monitoring gene targeting efficiency in human cell lines. (A) Representative flow cytometry results. The reporter and targeting vectors shown on the top were sequentially transduced into cells, and GFP-positive ratios were determined by fluorescence flow cytometry. Hereafter, AAV infection of the cells was performed at a multiplicity of infections of $1 \times 10^{4}$. FL1A (x-axis) and FL2A (y-axis) represent GFP signals and autofluorescence from cells, respectively. The GFP-positive ratios for the respective experiments are noted in the scatter plots. (B) Graphical display of all of the results $(n=3$; mean \pm SEM). (C) Southern blot analysis investigating the copy number of the Hyg ${ }^{R}-5^{\prime}$ EGFP reporter vector within the genome of each reporter clone. gDNA was extracted from parental DLD-1 (D) and HCT116 (H) cell lines (parent) as well as from Hyg ${ }^{\mathrm{R}} \mathrm{5}^{\prime}$ EGFP reporter clones (reporter) established from the parental cells, digested with either BamHI or Bg/ll, as noted below the gel image, and analyzed with a probe located on the $\mathrm{Hyg}^{\mathrm{R}}$ coding sequence as depicted in Figure 1 . Single bands detectable in Hyg $-5^{\prime}$ EGFP reporter clones are marked with white arrowheads. The positions of DNA size standards are shown to the right. HygR, hygromycin resistance gene; TV, targeting vector.

(PBS), and analyzed with a FACSCanto II flow cytometer (BD Biosciences). Cell sorting was performed with a FACSVantage SEM instrument (BD Biosciences).

Polymerase chain reaction

PCR was performed using Platinum Taq (Life Technologies) or Pwo DNA Polymerase (Roche, Basel, Switzerland) and a GeneAmp 9700 thermal cycler (Life Technologies). Genomic DNA (gDNA) for PCR templates was extracted using PureLink Genomic DNA Mini kit (Life Technologies) according to the manufacturer's instructions. To verify homologous integration of the targeting vector into the genome, PCR was performed using the following oligonucleotide primers: F1, 5'-TGTGTAGAAGTACTCGCCGA-3'; $\mathrm{R} 1$, 5'-TCCAGCAGGACCATGTGATC-3'; F2, 5'-ACCATCTTCTTCAAGGACGA-3'; and R2, 5'AGTACTCACCCCAACAGCTG-3'.
Southern blot analysis

For Southern blot analyses, gDNA was extracted using standard phenol/chloroform extraction and ethanol precipitation. Five micrograms gDNA were digested with the appropriate restriction enzymes, fractionated on $0.7 \%$ agarose gels, and blotted onto Hybond-N+ membrane (GE Healthcare, Piscataway, NJ, USA). Probes were labeled using AlkPhos Direct Labeling and Detection System with CDP-Star kit (GE Healthcare) and used for hybridization.

Statistical analyses

Statistical analyses were performed using Intercooled Stata (Stata, College Station, TX, USA). The efficiency of establishing reporter clones was analyzed with Fisher's exact test. Two-way factorial analysis of variance was applied for the comparison of plasmid-based versus $A A V$-based targeting vectors and ATG-less TV versus SV40p TV after log trans- formation of the data. A paired $t$-test was used to compare the short and long $5^{\prime}$ arms.

\section{Results and discussion}

We initially constructed a reporter vector, the $\mathrm{Hyg}^{\mathrm{R}}-5^{\prime}$ EGFP reporter, carrying a fusion gene consisting of full-length $\mathrm{Hyg}^{\mathrm{R}}\left(5^{\prime}\right.$ side) and a $5^{\prime}$ portion of EGFP (500 bp in length; $3^{\prime}$ side) fused to each other in a single frame (Figure 1). We also constructed a targeting vector, ATG-less TV (Figure 1), containing an EGFP gene without an authentic start codon to reconstitute functional $\mathrm{EGFP}$ via $\mathrm{HR}$ with the $\mathrm{Hyg}^{\mathrm{R}}-5^{\prime}$ EGFP fusion gene in the reporter vector. ATG-less TV uses a promoter-trap strategy for efficient gene targeting $(18,19)$ and thus has no promoter to drive $\mathrm{Neo}^{\mathrm{R}}$ expression. In addition, ATG-less TV is constructed with an AAV backbone, because AAV-based targeting vectors achieve more than 1000-fold higher rates of gene targeting than conventional plasmid-based targeting vectors (5).

When cells are stably transduced with the $\mathrm{Hyg}^{\mathrm{R}}-5^{\prime}$ EGFP reporter vector and the ATG-less TV, gene-targeting events are detectable by a green fluorescent signal. Because the $H / R$ ratio of targeting vectors is one of the most critical determinants of the difficulty of gene targeting, we addressed the ability of this system to assay $\mathrm{H} / \mathrm{R}$ ratios. We first transfected the $\mathrm{Hyg}^{\mathrm{R}}-5^{\prime}$ EGFP reporter vector into the HCT116 and DLD-1 colon cancer cell lines, and isolated single cell clones through hygromycin selection (hereafter referred to as reporter clones). The resulting six reporter clones from HCT 116 and six from DLD-1 were then infected with ATG-less TV and selected with G418. Fluorescence flow cytometric analyses of the resulting bulk cell populations exhibited subsets of GFP-positive cells in one reporter clone from each cell line (Figure 2, A and B, and Table 1). These results strongly suggested that homologous regions within the two vectors were recombined in subsets of cells in these clones, leading to the production of functional GFP. We also confirmed the absence of appreciable GFP-positive cell populations within parental HCT116 and DLD-1 cells as well as in cells transduced with either the $\mathrm{Hyg}^{\mathrm{R}}-5^{\prime}$ EGFP reporter or ATG-less TV alone. Southern blot analysis of a functional reporter clone from each of these cell lines demonstrated that these reporter clones are likely to harbor single copies of the $\mathrm{Hyg}^{\mathrm{R}}-5^{\prime}$ EGFP reporter vector in the genome (Figure 2C).

In the other five reporter clones for each cell line, no GFP-positive cell populations were detected by flow cytometry after infection with ATG-less TV. Although the reason for this is unclear, it may be possible that the location of the $\mathrm{Hyg}^{\mathrm{R}}-5^{\prime}$ EGFP reporter vector within the genome affects the efficiency of $\mathrm{HR}$ 
with ATG-less TV. Another possibility is that all or part of the $3^{\prime}$ arm of the $\mathrm{Hyg}^{\mathrm{R}}-5^{\prime}$ EGFP reporter vector may be subjected to terminal deletion upon integration into the genome, which precludes gene targeting by ATG-less TV.

We next carried out cell sorting of an HCT116-derived reporter clone transduced with ATG-less TV and isolated GFP-positive and -negative populations. A total of 15 single cell clones were established from the respective populations and analyzed by PCR to determine whether GFP-positive cells had truly undergone gene targeting by ATG-less TV. PCR over the $5^{\prime}$ arm of ATG-less TV (F1-R1; Figure 3, A and B) indicated that gene targeting was achieved in at least 14 of the 15 single cell clones isolated from the GFP-positive cell population, whereas none of the clones from the GFP-negative population underwent gene targeting. We also confirmed the reconstitution of a full-length $\mathrm{Hyg}^{\mathrm{R}}$-EGFP gene in a few GFP-positive single cell clones by DNA sequencing (data not shown). In addition, we carried out Southern blot analyses of several GFP-positive and -negative single cell clones derived from the HCT116 and DLD-1 cell lines, and further confirmed that ATG-less $\mathrm{TV}$ was homologously recombined with the $\mathrm{Hyg}^{\mathrm{R}}-5^{\prime}$ EGFP reporter vector within the genome of GFP-positive cells (Figure 3C). These results collectively indicate that GFP positivity in the $\mathrm{Hyg}^{\mathrm{R}}-5^{\prime}$ EGFP reporter system represents gene targeting events.

To determine the accuracy of the $\mathrm{Hyg}^{\mathrm{R}}-5^{\prime}$ EGFP reporter system for monitoring gene targeting efficiency, we subsequently investigated whether this reporter system could reproduce previous findings of gene targeting efficiency achieved with various types of targeting vectors. We first infected (as an AAV-based targeting vector) or transfected (as a plasmid-based targeting vector) HCT116 and DLD-1-derived $\mathrm{Hyg}^{\mathrm{R}}{ }^{-} \mathrm{5}^{\prime}$ EGFP reporter clones with ATG-less TV and then selected the cells with G418. Flow cytometric analyses demonstrated that AAV-based ATG-less TV infection achieved an average of $>100$-fold gene targeting efficiency compared with that of the plasmid version of ATG-less TV (Figure 4A), reproducing previous results $(5,6,16)$. We also addressed the impact of the promoter-trap strategy on gene targeting efficiency in this system. To this end, we constructed an AAV-based targeting vector similar to ATG-less TV, except that it lacked a synthetic intron and IRES, containing a poly(A) site and an SV40 promoter to drive $\mathrm{Neo}^{\mathrm{R}}$ instead, thus no longer supporting the promoter-trap strategy (SV40p TV; Figure 1). Fluorescence flow cytometric analyses demonstrated that the infection of $\mathrm{Hyg}^{\mathrm{R}}-5^{\prime}$ EGFP reporter cell clones with ATG-less TV achieved, on average, 40 -fold greater gene
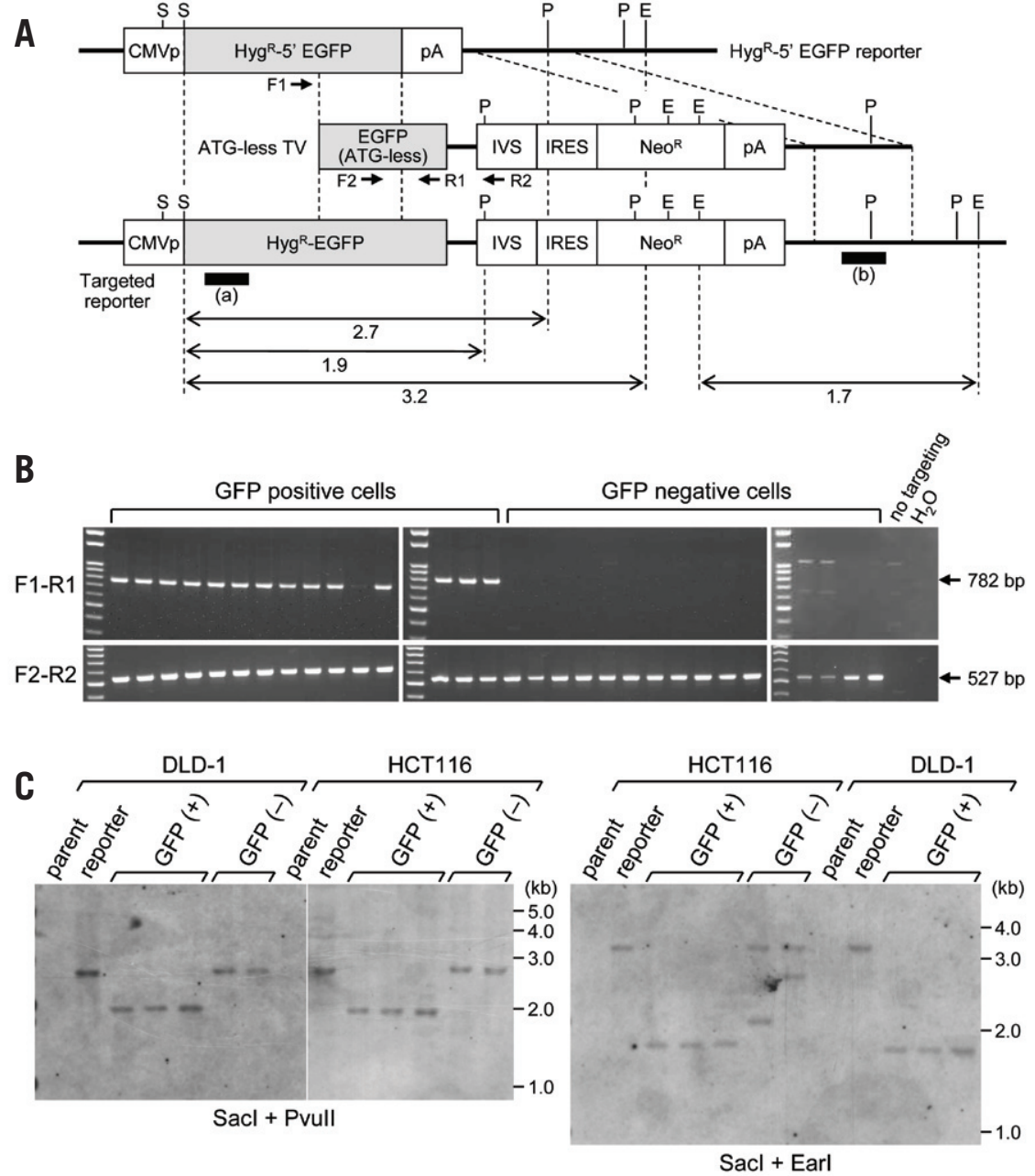

Figure 3. Confirmatory assays demonstrating that GFP-positive clones were generated by gene targeting. (A) The Hyg $-5^{\prime}$ EGFP reporter vector, ATG-less TV, and a product of homologous recombination between these vectors. F1, F2, R1, and R2 indicate the location of the primers used in the PCR shown in panel B. Filled rectangles indicate the location of probes on $\mathrm{Hyg}^{\mathrm{R}}$ (a) and partial SV40 large T (b) genes used in the Southern blot analyses shown in panel C. Restriction enzyme sites were abbreviated as follows and marked on the diagrams: S, Sacl; P, Pvull; E, Earl. Arrows at the bottom show the distances between the restriction enzyme sites in kilobase pairs. The diagrams are not drawn to scale. (B) PCR over the $5^{\prime}$ homology arm of ATGless TV. The HCT116-derived Hyg $-5^{\prime}$ EGFP reporter cell clones infected with ATG-less TV were sorted into GFP-positive and -negative populations by flow cytometry. A total of 15 single cell clones from each of the populations was expanded and analyzed by PCR. The size of the PCR amplicons is denoted to the right. Note that a faint band seen in the 11th lane from the left on the F1-F2 gel image was determined as being marginally positive. PCR primers F1, R1, F2, and R2 are depicted in panel A. No targeting: a reporter clone not infected with the targeting vector. (C) Southern blot analyses of GFP-positive and -negative single cell clones. Several DLD-1-derived and HCT116-derived single cell clones were established as in panel B, and gDNA from these clones was digested with the restriction enzymes indicated below the gel images. Probes (a) and (b) depicted in panel A were used for Southern hybridization with Sacl/Pvull-digested gDNA and Sacl/Earl-digested gDNA, respectively. Note that probing for $\mathrm{Hyg}^{\mathrm{R}}$ and SV4O large T sequences leads to the detection of one and two bands, respectively, in Hyg ${ }^{\mathrm{R}} 5^{\prime}$ EGFP reporter clones with random integration of ATG-less TV into the genome. The positions of the DNA size standards are shown to the right of each image.

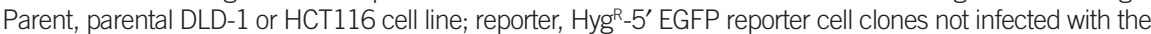
targeting vector. Hyg ${ }^{\mathrm{R}}$, hygromycin resistance gene; TV, targeting vector; CMVp, cytomegalovirus promoter; IVS, synthetic intron; IRES, internal ribosomal entry site; $\mathrm{Neo}^{\mathrm{R}}$, neomycin resistance gene; pA, poly(A) sites.

targeting efficiency than SV40p TV (Figure $4 \mathrm{~B}$ ), which is similar to the results reported by previous studies $(6,16)$. Increasing homology length is also likely to increase targeting rates (11). We thus shortened the $5^{\prime}$ homology arm of ATG-less TV and examined its impact on gene targeting efficiency. The $5^{\prime}$ homology arm of ATG-less TV is 719 bp in length, and a 113-bp fragment at its $5^{\prime}$ terminus was deleted to create another targeting vector, $3^{\prime}$ EGFPTV(Figure 1). This truncation resulted in a reproducible decrease in gene targeting efficiency, although our results did not reach statistical significance (Figure 4C). Overall, our $\mathrm{Hyg}^{\mathrm{R}}-5^{\prime}$ EGFP reporter system successfully reproduced the impact of targeting vector 


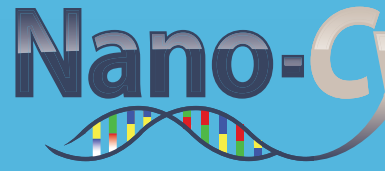

eliminates microscope drift!

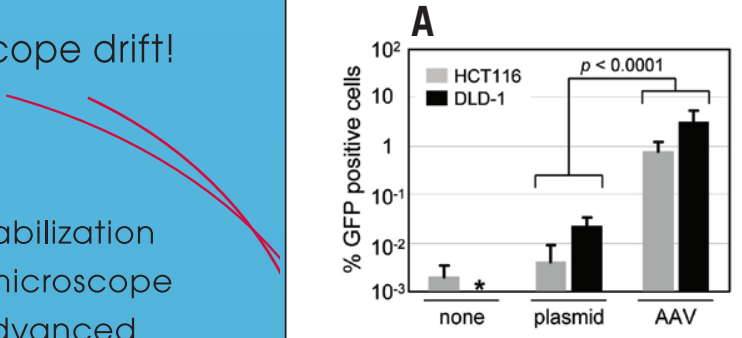

The Nano-Cyte stabilization

system eliminates microscope

drift that limits advanced

fluorescence imaging methods.

The Nano-Cyte uses 3D

localization information from the acquired image to provide

active position changes to the sample therefore stabilizing the image.

Nano-Cyte patented technology corrects drift in all 3 dimensions to within $\pm 10 \mathrm{~nm}(X Y)$ and $\pm 20 \mathrm{~nm}(Z)$.

Nano-Cyte can correct for drift over a period of days enabling long term imaging.

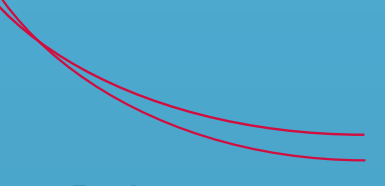

\section{Features}

- Eliminates microscope drift

- Image stabilization for days

- 3D stabilization at the nanometer scale

- Simultaneous image acquisition and stabilization

- Integrated hardware with automated software control Discover more at

Neuroscience 2012, Booth 830

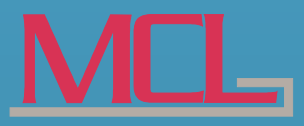

USA: sales@madcitylabs.com

Europe: sales@madcitylabs.eu www.madcitylabs.com
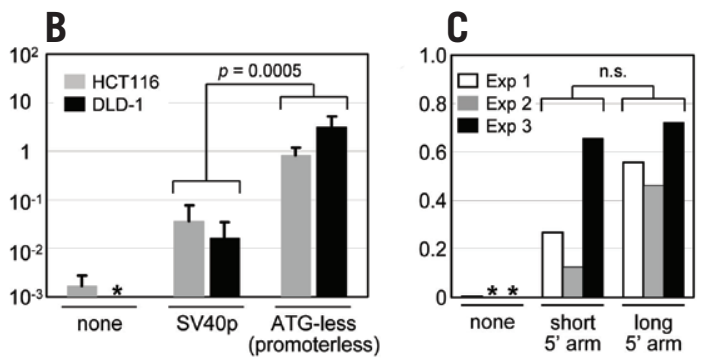

Figure 4. The $\mathrm{Hyg}^{\mathrm{R}}-5^{\prime}$ EGFP reporter system monitors the efficiency of gene targeting performed by various targeting vectors. The Hyg ${ }^{\mathrm{R}} 5^{\prime}$ EGFP reporter clones were transduced with the targeting vectors denoted at the bottom of each panel, selected with G418, and analyzed by fluorescence flow cytometry. (A) Reporter clones were either transfected (as a plasmid) or infected (as an AAV) with ATG-less TV ( $n=3$; mean \pm SEM). (B) Reporter clones were infected with AAV-based targeting vectors with (ATG-less TV) or without (SV40p TV) a promoter-trap system ( $n=3$; mean \pm SEM). (C) A HCT116derived reporter clone was infected with targeting vectors containing $5^{\prime}$ homology arms that were 606 bp (3' EGFP TV; short 5' arm) or 719 bp (ATG-less TV; long 5' arm) in length. For panels A-C, uninfected reporter clones were used as controls. Asterisks indicate a GFP-positive ratio of $0 \%$.

designs on gene targeting efficiency described in previous studies.

To clarify the advantage of the use of our $\mathrm{Hyg}^{\mathrm{R}}-5^{\prime}$ EGFP reporter system in monitoring gene targeting efficiency, we next compared the efficiency of generating reporter clones in our Hyg ${ }^{\mathrm{R}}-5^{\prime}$ EGFP system with that of a conventional reporter system that uses an unfused $5^{\prime}$ EGFP gene as a reporter ( $5^{\prime}$ EGFP reporter). We first isolated multiple HCT116-derived cell clones transduced with the $5^{\prime}$ EGFP reporter vector and infected the clones with ATG-less TV. The resulting bulk population of G418-resistant cells from each 5' EGFP reporter clone was then analyzed by fluorescence flow cytometry, similar to that for the $\mathrm{Hyg}^{\mathrm{R}}-5^{\prime}$ EGFP reporter clones. It appeared that functional reporter clones were obtained with higher efficiency by the $\mathrm{Hyg}^{\mathrm{R}}-5^{\prime}$ EGFP reporter system than that with the $5^{\prime}$ EGFP reporter system (Table 1), although the difference did not reach statistical significance presumably because of the limited number of cell clones analyzed. Southern blot analysis demonstrated that the two functional $5^{\prime}$ EGFP reporter clones are likely to carry single $5^{\prime}$ EGFP reporter vectors within the genome (data not shown).

We also addressed the ability of reporter cell clones in the Hyg ${ }^{\mathrm{R}} 5^{\prime}$ EGFP and 5' EGFP reporter systems to monitor gene targeting efficiency over time. Reporter clones bearing functional $\mathrm{Hyg}^{\mathrm{R}}-5^{\prime} \mathrm{EGFP}$ or $5^{\prime} \mathrm{EGFP}$ reporter genes were maintained in culture for long durations (no less than 4 weeks), infected with ATG-less TV, selected with G418, and then analyzed by fluorescence flow cytometry. GFP-positive ratios in individual reporter clones were then compared with those obtained before the above mentioned long-term culture. We found that GFP-positive ratios before and after the long-term culture were equivalent in the $\mathrm{Hyg}^{\mathrm{R}}-5^{\prime}$ EGFP reporter clones; however, in $5^{\prime}$ EGFP reporter clones, GFP-positive ratios were dramatically decreased after the long-term culture (Figure 5). These data demonstrated that our $\mathrm{Hyg}^{\mathrm{R}}-5^{\prime}$ EGFP reporter system provides a prolonged capacity to monitor gene targeting efficiency relative to a conventional reporter system.

In this study, we developed a molecular system to monitor gene targeting efficiency in human somatic cell lines. This system uses the Hyg ${ }^{R}$-EGFP fusion gene for a dual purpose, namely, antibiotic selection as well as fluorescence-based monitoring of gene targeting events. When a reporter gene is expressed independent of an antibiotic resistance gene, as in conventional reporter vectors, cell clones transduced with such vectors rarely serve as potent reporter systems (Table 1). Moreover, reporter cell clones bearing such reporter vectors are prone to lose their function over time during cell culture (Figure 5). In contrast, the use of the $\mathrm{Hyg}^{\mathrm{R}}$-EGFP fusion gene allows the establishment of potent reporter clones in a less labor-intensive fashion, and the resultant reporter clones retain their function over a long duration. Indeed, the $\mathrm{Hyg}^{\mathrm{R}}-5^{\prime}$ EGFP reporter clones were functional throughout the study period of more than 6 months.

Another strategy permitting the acquisition of potent reporter clones in an efficient fashion may be the use of IRES to join a selection marker gene and a reporter gene instead of directly fusing them (12). By maintaining reporter clones in a selective condition, this type of reporter system may also ensure robust, prolonged expression of reporter genes. However, cistrons joined by IRES do not always produce an equivalent level of proteins. It has been shown that the amounts of two proteins produced from a transcript containing IRES vary in a positiondependent fashion (20), as well as by host cell type (21,22), presumably because translation downstream of IRES is modulated by multiple regulatory factors $(23,24)$. In contrast, a fusion protein in our system secures the production of exactly the same amount of antibiotic resis- 


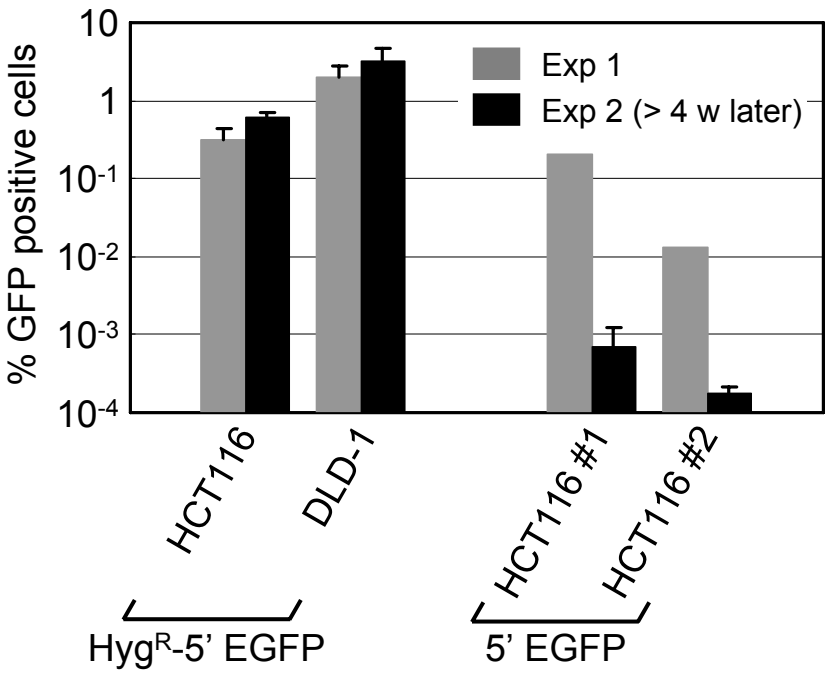

Figure 5. Use of the HygR ${ }^{\mathrm{R}} \mathrm{5}^{\prime}$ EGFP gene ensures robust and prolonged reporter activity. The gene targeting efficiency of the reporter clones denoted at the bottom of the figure was assayed twice with an interval of no less than 4 weeks. In each assay, reporter clones were transduced with ATG-less TV, selected with G418, and analyzed by flow cytometry to quantify GFP signals. Analyses included one each of the HCT116 and DLD-1-derived Hyg ${ }^{\mathrm{R}} 5^{\prime}$ EGFP reporter clones as well as two HCT116-derived 5' EGFP reporter clones. The second assays were performed on the 82nd (HCT116, HygR-5' EGFP), 69th (DLD-1, Hyg ${ }^{\mathrm{R}}$ 5' $^{\prime}$ EGFP), 28th (HCT116\#1, 5' EGFP), and 36th (HCT116\#2, 5' EGFP) day after the first assays. The first assays for the 5' EGFP reporter clones were performed without multiplication and thus have no error bars. The other assays were performed in triplicate (mean \pm SEM).

tance and reporter proteins, thus offering more stable activity as a reporter.

Another strategy to prevent attenuation of reporter gene function is the use of an endogenous gene as a reporter. One of the endogenous genes most commonly used for this purpose is the hypoxanthine phosphoribosyltransferase 1 (HPRTI) gene on the $\mathrm{X}$ chromosome. In cells bearing a single $H P R T 1$, such as diploid cells of male origin, the disruption of single HPRT1 alleles results in the loss of hypoxanthine phosphoribosyltransferase, which is selectable with an anti-metabolic nucleoside analog 6-thioguanine (6-TG) $(5,25)$. Furthermore, targeted correction of mutant HPRT1 can be detected by culturing cells with hypoxanthine-aminopterin-thymidine (HAT) medium $(25,26)$. As a drawback, however, this system cannot be utilized with cells bearing multiple $\mathrm{X}$ chromosomes, such as female cells and majority of aneuploid cells. In addition, cells exhibiting the microsatellite instability (MIN) phenotype are generally resistant to 6-TG (27) and are thus not eligible for HPRT1 targeting followed by 6-TG selection. In contrast, our reporter system is suitable for virtually any type of cell of male or female origin, including those with a MIN phenotype. Furthermore, our system determines gene targeting efficiency through rapid, easily performed high-throughput procedures with the aid of flow cytometry, unlike the HPRT1-based system in which gene targeting efficiency is estimated by counting colonies on tissue culture plates after incubation of the cells with and without 6-TG.
Besides determining the $\mathrm{H} / \mathrm{R}$ ratio of targeting vectors, the $\mathrm{Hyg}^{\mathrm{R}}-5^{\prime}$ EGFP system permits estimation of the absolute gene targeting frequency. This is accomplished by counting colonies formed on tissue culture plates after antibiotic selection in parallel with flow cytometric analyses of antibiotic-resistant cells. The absolute gene targeting frequency can be estimated by multiplying the number of colonies on the plate by the GFP-positive ratio determined by flow cytometry and then dividing the resulting number by the number of cells prepared for infection of the targeting vector.

Although gene targeting in human somatic cells can be achieved by several different methodologies (12,28-30), AAV-mediated gene targeting has an advantage in that it does not actively introduce DNA doublestrand breaks, thus likely generating fewer undesired alterations in the genome. Because the technology of AAV-mediated gene targeting is simple, cost-sensitive, publicly available, and capable of modifying human somatic cell genomes with decent efficiency, it continues to be an important alternative methodology for genome editing in human cell lines. Indeed, $\mathrm{AAV}$-mediated gene targeting has been utilized in many recent studies modifying genomes in human cell lines with the aim of gene functional analyses $(4,17,31-$ 38). As shown in this study, our $\mathrm{Hyg}^{\mathrm{R}}-5^{\prime}$ EGFP reporter system enables robust monitoring of the efficiency of AAV-mediated gene targeting in human somatic cells. This reporter system will thus prove helpful in improving the design of AAV-based targeting vectors and optimizing experimental conditions for gene targeting. It may also assist in screening for genes and drugs that affect the incidence of $\mathrm{HR}$, potentially leading to further improvements in gene targeting technology. Such technical improvements will eventually result in the generation of highly efficient gene targeting systems readily applicable to various types of human cells.

\section{Acknowledgments}

The authors are thankful to Drs. Ben H. Park and Fred Bunz (Johns Hopkins University) for providing the reagents. This work was partly supported by Grants-in-Aid for Scientific Research from the Japan Society for the Promotion of Science (nos. 22700888 to S.K., 21591225 to Y.H., and 22500999 to H.K.), Strategic Research Foundation Grant-aided Project for Private Universities from the Ministry of Education, Culture, Sports, Science, and Technology (MEXT), Japan (S1101027 to S.K., Y.H., and H.K.), Aichi Cancer Research Foundation (to S.K., Y.H., and H.K.), and AIKEIKAI Foundation (to S.K.).

\section{Competing interests}

The authors declare no competing interests.

\section{References}

1. Shirasawa, S., M. Furuse, N. Yokoyama, and T. Sasazuki. 1993. Altered growth of human colon cancer cell lines disrupted at activated Ki-ras. Science 260:85-88.

2. Sedivy, J.M., B. Vogelstein, H.L. Liber, E.A. Hendrickson, and A. Rosmarin. 1999. Gene targeting in human cells without isogenic DNA. Science 283:9a.

3. Di Nicolantonio, F., S. Arena, M. Gallicchio, D. Zecchin, M. Martini, S.E. Flonta, G.M.Stella, S. Lamba, et al. 2008. Replacement of normal with mutant alleles in the genome of normal human cells unveils mutation-specific drug responses. Proc. Natl. Acad. Sci. USA 105:20864-20869.

4. Di Nicolantonio, F., S. Arena, J. Tabernero, S. Grosso, F. Molinari, T. Macarulla, M. Russo, C. Cancelliere, et al. 2010. Deregulation of the PI3K and KRAS signaling pathways in human cancer cells determines their response to everolimus. J. Clin. Invest. 120:2858-2866.

5. Russell, D.W. and R.K. Hirata. 1998. Human gene targeting by viral vectors. Nat. Genet. 18:325330.

6. Hirata, R., J. Chamberlain, R. Dong, and D.W. Russell. 2002. Targeted transgene insertion into human chromosomes by adeno-associated virus vectors. Nat. Biotechnol. 20:735-738.

7. Rago, C., B. Vogelstein, and F. Bunz. 2007. Genetic knockouts and knockins in human somatic cells. Nat. Protocols 2:2734-2746.

8. Konishi, H., J. Lauring, J.P. Garay, B. Karakas, A.M. Abukhdeir, J.P. Gustin, Y. Konishi, and B.H. Park. 2007. A PCR-based high-throughput screen with multiround sample pooling: application to somatic cell gene targeting. Nat. Protocols 2:2865-2874. 
BRAND Handystep ${ }^{\circledR} \mathcal{S}$ Repeating Pipette

The NEW HandyStep ${ }^{\circledR} \mathcal{S}$ provides unprecedented versatility in a mechanical repeating pipette, with accuracy, precision and dependability.

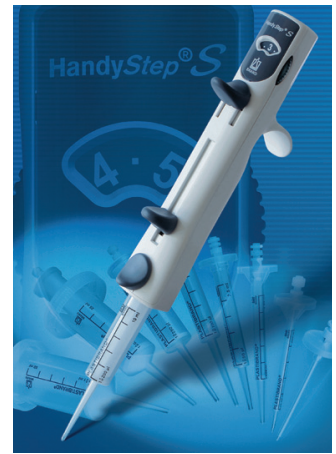

- Comfortable, light weight, and well balanced

- Easy one handed volume selection

- Flexible; accepts third party tips - no more being locked into a sole source

- Tip ejection system allows tip removal without user handling.

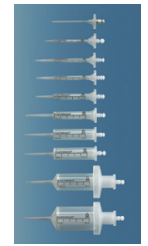

\section{Economical}

BRAND PD-Tip ${ }^{T M}$

syringe tips fit all

repeating pipettes

using non-proprietary designs.

Looking for an electronic repeating pipette? Try the rapid, relaxed and reliable HandyStep ${ }^{\circledR}$ electronic.

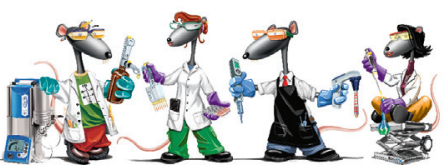

Lab Rats Trust BrandTech ${ }^{\circledR !}$

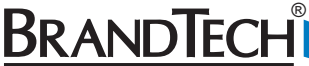
SCIENTIFIC, INC.
9. Khan, I.F., R.K. Hirata, and D.W. Russell. 2011 AAV-mediated gene targeting methods for human cells. Nat. Protocols 6:482-501.

10. Arbones, M.L., H.A. Austin, D.J. Capon, and G. Greenburg. 1994. Gene targeting in normal somatic cells: inactivation of the interferon-gamma receptor in myoblasts. Nat. Genet. 6:90-97.

11. Hirata, R.K. and D.W. Russell. 2000. Design and packaging of adeno-associated virus gene targeting vectors. J. Virol. 74:4612-4620.

26. Ohbayashi, F., M.A. Balamotis, A. Kishimoto, E. Aizawa, A. Diaz, P. Hasty, F.L. Graham, C.T. Caskey, and K. Mitani. 2005. Correction of chromosomal mutation and random integration in embryonic stem cells with helper-dependent adenoviral vectors. Proc. Natl. Acad. Sci. USA 102:13628-13633.

27. Swann, P.F., T.R. Waters, D.C. Moulton, Y.Z. Xu, Q. Zheng, M. Edwards, and R. Mace. 1996. Role of postreplicative DNA mismatch repair in the cytotoxic action of thioguanine. Science 273:1109-1111. nucleases stimulate gene targeting in human cells. Science 300:763.

13. Teschendorf, C., K.H. Warrington, Jr., D.W. Siemann, and N. Muzyczka. 2002. Comparison of the EF-1 alpha and the CMV promoter for engineering stable tumor cell lines using recombinant adeno-associated virus. Anticancer Res. 22:3325-3330.

14. Gopalkrishnan, R.V., K.A. Christiansen, N.I. Goldstein, R.A. DePinho, and P.B. Fisher. 1999. Use of the human EF-1alpha promoter for expression can significantly increase success in establishing stable cell lines with consistent expression: a study using the tetracycline-inducible system in human cancer cells. Nucleic Acids Res. 27:4775-4782.

15. Najjar, S.M. and R.E. Lewis. 1999. Persistent expression of foreign genes in cultured hepatocytes: expression vectors. Gene 230:41-45.

16. Topaloglu, O., P.J. Hurley, O. Yildirim, C.I. Civin, and F. Bunz. 2005. Improved methods for the generation of human gene knockout and knockin cell lines. Nucleic Acids Res. 33:e158.

17. Konishi, H., B. Karakas, A.M. Abukhdeir, J. Lauring, J.P. Gustin, J.P. Garay, Y. Konishi, E. Gallmeier, et al. 2007. Knock-in of mutant K-ras in nontumorigenic human epithelial cells as a new model for studying K-ras mediated transformation. Cancer Res. 67:8460-8467.

18. Jasin, M. and P. Berg. 1988. Homologous integration in mammalian cells without target gene selection. Genes Dev. 2:1353-1363.

19. Sedivy, J.M. and P.A. Sharp. 1989. Positive genetic selection for gene disruption in mammalian cells by homologous recombination. Proc. Natl. Acad. Sci. USA 86:227-231.

20. Hennecke, M., M. Kwissa, K. Metzger, A. Oumard, A. Kroger, R. Schirmbeck, J. Reimann, and H. Hauser. 2001. Composition and arrangement of genes define the strength of IRES-driven translation in bicistronic mRNAs. Nucleic Acids Res. 29:3327-3334.

21. Borman, A.M., P. Le Mercier, M. Girard, and K.M. Kean. 1997. Comparison of picornaviral IRES-driven internal initiation of translation in cultured cells of different origins. Nucleic Acids Res. 25:925-932.

22. Roberts, L.O., R.A. Seamons, and G.J. Belsham. 1998. Recognition of picornavirus internal ribosome entry sites within cells; influence of cellular and viral proteins. RNA 4:520-529.

23. Filbin, M.E. and J.S. Kieft. 2009. Toward a structural understanding of IRES RNA function. Curr. Opin. Struct. Biol. 19:267-276.

24. Komar, A.A. and M. Hatzoglou. 2011. Cellular IRES-mediated translation: the war of ITAFs in pathophysiological states. Cell Cycle 10:229240.

25. So, S., Y. Nomura, N. Adachi, Y. Kobayashi, T. Hori, Y. Kurihara, and H. Koyama. 2006. Enhanced gene targeting efficiency by siRNA that silences the expression of the Bloom syndrome gene in human cells. Genes Cells 11:363-371.
28. Carroll, D. 2011. Zinc-finger nucleases: a panoramic view. Curr. Gene Ther. 11:2-10.

29. Miller, J.C., S. Tan, G. Qiao, K.A. Barlow, J. Wang, D.F. Xia, X. Meng, D.E. Paschon, et al. 2011. A TALE nuclease architecture for efficient genome editing. Nat. Biotechnol. 29:143-148.

30. Mussolino, C. and T. Cathomen. 2012. TALE nucleases: tailored genome engineering made easy. Curr. Opin. Biotechnol. Feb 17. [Epub ahead of print]

31. Hucl, T., C. Rago, E. Gallmeier, J.R. Brody, M. Gorospe, and S.E. Kern. 2008. A syngeneic variance library for functional annotation of human variation: application to BRCA2. Cancer Res. 68:5023-5030.

32. Gustin, J.P., B. Karakas, M.B. Weiss, A.M. Abukhdeir, J. Lauring, J.P. Garay, D. Cosgrove, A. Tamaki, et al. 2009. Knockin of mutant PIK3CA activates multiple oncogenic pathways. Proc. Natl. Acad. Sci. USA 106:2835-2840.

33. Yun, J., C. Rago, I. Cheong, R. Pagliarini, P. Angenendt, H. Rajagopalan, K. Schmidt, J.K. Willson, et al. 2009. Glucose deprivation contributes to the development of KRAS pathway mutations in tumor cells. Science 325:1555-1559.

34. Vitolo, M.I., M.B. Weiss, M. Szmacinski, K. Tahir, T. Waldman, B.H. Park, S.S. Martin, D.J. Weber, and K.E. Bachman. 2009. Deletion of PTEN promotes tumorigenic signaling, resistance to anoikis, and altered response to chemotherapeutic agents in human mammary epithelial cells. Cancer Res. 69:8275-8283.

35. Chung, J.H. and F. Bunz. 2010. Cdk2 is required for p53-independent G2/M checkpoint control. PLoS Genet. 6:e1000863.

36. Fattah, F., E.H. Lee, N. Weisensel, Y. Wang, N. Lichter, and E.A. Hendrickson. 2010. Ku regulates the non-homologous end joining pathway choice of DNA double-strand break repair in human somatic cells. PLoS Genet. 6:e1000855.

37. Solomon, D.A., T. Kim, L.A. Diaz-Martinez, J. Fair, A.G. Elkahloun, B.T. Harris, J.A. Toretsky, S.A. Rosenberg, et al. 2011. Mutational inactivation of STAG2 causes aneuploidy in human cancer. Science 333:1039-1043.

38. Konishi, H., M. Mohseni, A. Tamaki, J.P. Garay, S. Croessmann, S. Karnan, A. Ota, H.Y. Wong, et al. 2011. Mutation of a single allele of the cancer susceptibility gene BRCA1 leads to genomic instability in human breast epithelial cells. Proc. Natl. Acad. Sci. USA 108:17773-17778.

Received 10 April 2012; accepted 25 July 2012.

Address correspondence to Hiroyuki Konishi, Department of Biochemistry, Aichi Medical University School of Medicine, 1-1 Yazako Karimata, Building \#2, Room 362, Nagakute, Aichi, Japan. Email: hkonishi@aichi-med-u.ac.jp

To purchase reprints of this article, contact: biotechniques@fosterprinting.com 